\title{
Acute spinal epidural hematoma, clinical and etiopathogenic diagnostic difficulties - Case presentation and synthesis of the literature
}

Aurelian Anghelescu ${ }^{1,2}$, Cristina Cîmpeanu ${ }^{1}$, Alina Oana Băjenaru' ${ }^{1}$, Ionut Octavian Frecea ${ }^{1}$, Daniel Șerban ${ }^{3}$, Gelu Onose ${ }^{1,2}$

Corresponding author: Aurelian Anghelescu, aurelian_anghelescu@yahoo.co.uk,

1. Neurorehabilitation Clinic, Teaching Emergency Hospital "Bagdasar-Arseni" (TEHBA), Bucharest, Romania

2. "Carol Davila" University of Medicine and Pharmacy, Bucharest, Romania 3. Spinal Neurosurgical department Teaching Emergency Hospital "Bagdasar-Arseni" (TEHBA), Bucharest, Romania

\begin{abstract}
Introduction: Epidural hematoma has a double anatomopathological topography: intracranial and/ or spinal. Its etiology is complex: post-traumatic (spinal trauma, or lumbar puncture), iatrogenic (secondary to an inadequate anticoagulation or antiplatelet treatment), congenital or acquired disorders of coagulation (leukemia, hepatic cirrhosis), secondary to intense Valsalva maneuvers (e.g. during labor, or an intense physical effort), and idiopathic.

The purpose of this article is to present a clinical case of acute spinal epidural hematoma (SEDH) with atypical clinical picture and a puzzled pathophysiological mechanism, and also a brief review of the relevant literature.

Case presentation: An 80-years-old male patient, with locomotor disability (bilateral congenital foot deformity), and multiple cardiovascular comorbidities (chronic atrial fibrillation (AF), dilated cardiomyopathy and contractile dysfunction (chronic heart failure, with left ventricle ejection fraction $40 \%$ ), chronically anticoagulated with a vitamin $\mathrm{K}$ antagonist (acenocumarol). The elderly submitted a body-level fall without cranial trauma, event followed by a short loss of consciousness (without convulsions or sphincter relaxation). He suffered a low-energy cervical fracture (C7 vertebral injury) and a posterolateral acute SEDH at C3-Th2 vertebral levels.

Decompressive hemilaminectomy at the C4-Th2 levels and evacuation of the SEDH, was performed during the early sub-acute phase. The patient was transferred in our rehabilitation clinic as C4 AIS-C tetraplegia (global motor score 50/100), neurogenic bladder and bowel, with post surgical wound dehiscence (healed per secundam). The subject had a favorable neurological evolution and was discharged as C7 AIS-D tetraplegia (global motor score 81/100).

Discussion: The case particularity consists in a puzzled etiopathogenetic mechanisms and difficulty to accurately indicate the chronological chain of events generating the acute SEDH.

An overdosed anticoagulant therapy might be incriminated as an iatrogenic cause for a "spontaneous" SEDH, but most probably its etiology is complex, probably traumatic, consequence of the cervical spine fracture due the low-intensity biomechanical impact.

The complex predisposing circumstances to accidental fall in our elderly patient were due to the:

- impaired, unstable locomotor function, secondary to his bilateral congenital clubfoot deformity / disability

- chronic AF, contractile dysfunction and hypodiastolic phenomena, with cardiogenic syncope and global brain ischemia or transient ischemic cerebral attack.

Despite the good immediate outcomes, his future functional prognosis might be poor, due to the advanced age, severe cardiovascular pathology and the complex disturbances of the neuro-myo-artro-kinetic apparatus (major impediments of the somatic / body functions and structure). This health-related condition had severe repercussions on the subject's activity (related to tasks and basic activities of daily living) and participation, affecting the outcome of rehabilitation, and his quality of life.

Conclusions: Clinicians should consider the remote risk of SEDH (even with atypical clinical presentation) in patients with AF and anticoagulant medication.

Despite a postponed decompressive intervention (imposed by the severe comorbidities), our patient neurologically improved without recurrence, following a complex neurorehabilitation program.
\end{abstract}

Key words: spinal epidural hematoma, elderly, same-level fall, tetraplegia, hemilaminectomy, rehabilitation,

Introduction Spinal epidural hematoma $(\mathrm{SEDH})$ is a rare but serious condition. Its incidence is one per million inhabitants [1], accounting for less than $1 \%(0.3 \%$ to $0.9 \%)$ of the spinal epidural spaceoccupying lesions [2], [3].

Epidural hematoma has a double anatomopathological topography: intracranial and/or spinal [4]. Any level of the spinal canal may be involved, but highest frequency of SEDH distribution is in the upper thoracic (Th3-5) and middle to low cervical regions (C4-6) [5], [6].

The majority of hematomas $(53 \%)$ is located posterior to the spinal cord, $23 \%$ are located posterolateral, $18 \%$ anterior, whereas $5 \%$ are lateral and $1 \%$ are circumferential to the cord[6].

Acute SEDH is a rare neurosurgical emergency most often associated with acute symptomatic spinal cord compression and potentially permanent 
neurologic deficits. Most patients present with sudden (radicular) back pain and paraplegia or tetraplegia (in variable degrees of severity), possibly associating neurogenic bladder [3],[4],[7],,[8],[9],[10].

It can cause severe morbidity and mortality in $5.7 \%$ of cases [11]. Any age groups can be affected, but most cases are in their 60 s or 70 s, with a slight predominance $(1.4: 1)$ of the male gender [2], [7], [8], [11], [12], [13].

Most cases of acute SEDH have a multifactorial etiology, including old age, uncontrolled arterial hypertension, congenital and acquired coagulopathies, platelet dysfunction or even anti-platelet medication (aspirin, clopidogrel) [14], anticoagulants [15], [16], vascular malformations, tumors, spinal surgery or spinal procedures (lumbar punctures, epidural anesthesia) [10], [17], severe hepato-renal comorbidities (cirrhosis, liver transplantation, hemodialysis) [10], [17], even intense efforts implying sustained Valsalva maneuvers (intense efforts, lifting heavy weights [8], childbirth).

The paraclinical diagnosis is performed mainly with magnetic resonance imaging (MRI) [8].

The management of acute SEDH usually implies a surgical solution: decompressive hemilaminectomy and hematoma evacuation. Conservative treatment is reserved for subjects with minimal neurological impairment or with high risk for surgery [5], [6], [8], [13], [14], [19].

In rapid deterioration of the neurological status the most appropriate management is emergency decompressive hemilaminectomy and hematoma evacuation [17]

Case report This is a retrospective case study of an 80-year-old man who fell from body level on the ischiadic regions and suffered a $\mathrm{C} 7$ vertebral fracture, and a painless cervical SEDH at C3-Th2 vertebral levels.

Complex comorbidities were present in his medical history:

- Bilateral congenital foot deformity (fig. 1)

- Dilated cardiomyopathy with decompensated heart failure (NYHA IV then III, left ventricular systolic dysfunction with $40 \%$ ejection fraction)

- Permanent AF, chronically anticoagulated with a vitamin $\mathrm{K}$ antagonist (VKA, acenocumarol)

- Degenerative, large aortic stenosis
- Stage 2 arterial hypertension and episodes of orthostatic hypotension

- Dyslipidemia

- Cerebral microangipathy, vertebro-basilar circulatory insufficiency

- Cervical herniated disc C3-C4 (MRI scan performed on 08.12.2017)

- Chronic alcoholism

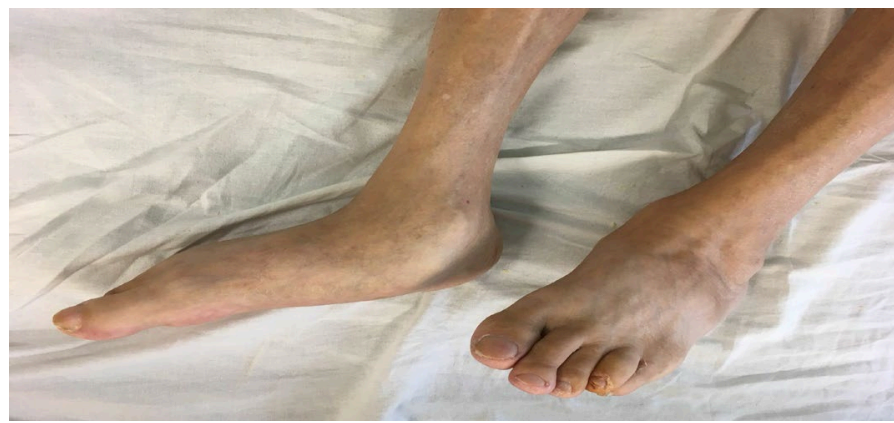

Fig.1 Bilateral congenital foot deformity

The elderly felt dizzy a few days before, and on 26.11.2018 he had accidentally fallen (from body level) on his ischial tuberosities, without cranial impact with the surrounding objects (e.g. furniture). This episode was followed by a short loss of consciousness (without convulsions or sphincter relaxation). When he recovered consciousness he accused motor limbs deficit, mainly on the left side, and neurogenic bladder.

The patient was first admitted in the Cardiology Clinic of Fundeni Hospital (28.11.2017 08.12.2017). CT scan of the brain (29.11.2017) ruled out acute ischemic or hemorrhagic lesions and revealed cerebral microangipathy (Fig. 2).

The patient was transferred in the neurosurgical spinal department of THEBA (on 08.12.2017, eleven days after the onset of symptoms), as C4 AIS-C tetraplegia. Spinal MRI scan revealed a posterior epidural hematoma in the cervico-thoracic region, compressing the cervical spinal cord, and $\mathrm{C} 7$ fracture (body and vertebral arch) - fig.3. MRI angiography revealed hypoplasia of the right vertebral artery.

The patient underwent a surgical decompressive intervention (on 13.12.2017): hemilaminectomy at the $\mathrm{C} 4-\mathrm{Th} 2$ levels, and evacuation of the hematoma (fig.4). The $\mathrm{C} 7$ vertebral fracture was conservatory treated (external immobilization with Philadelphia collar). 


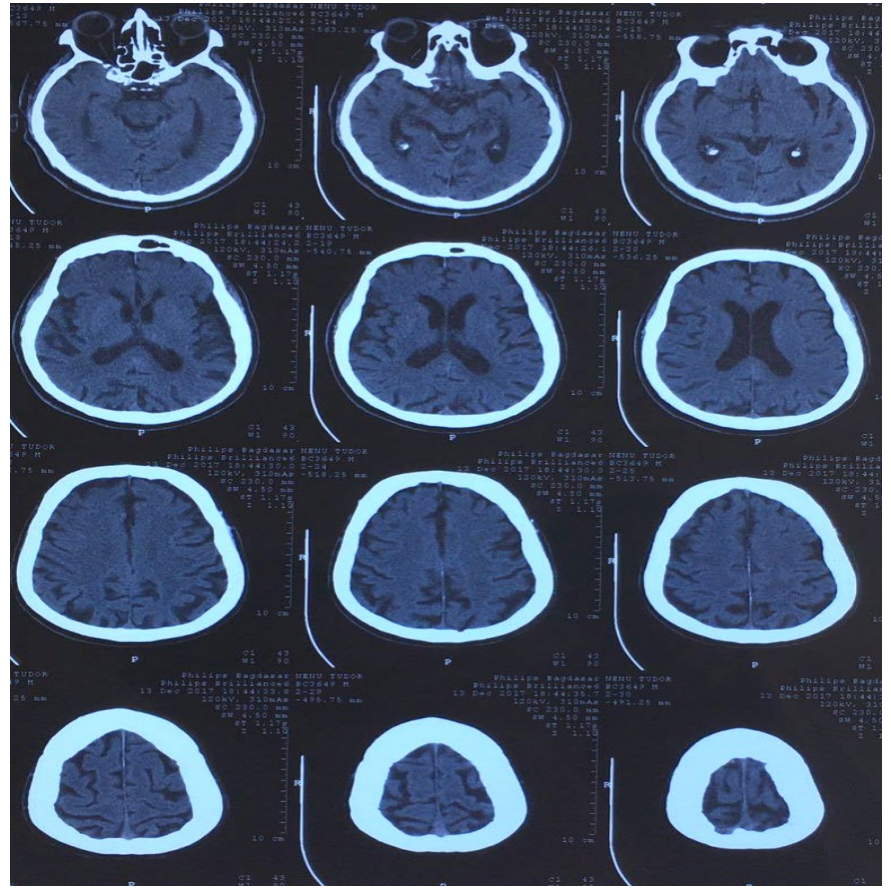

Fig.2 CT cerebral scan. Neither ischemic nor hemorrhagic acute / subacute lesions.

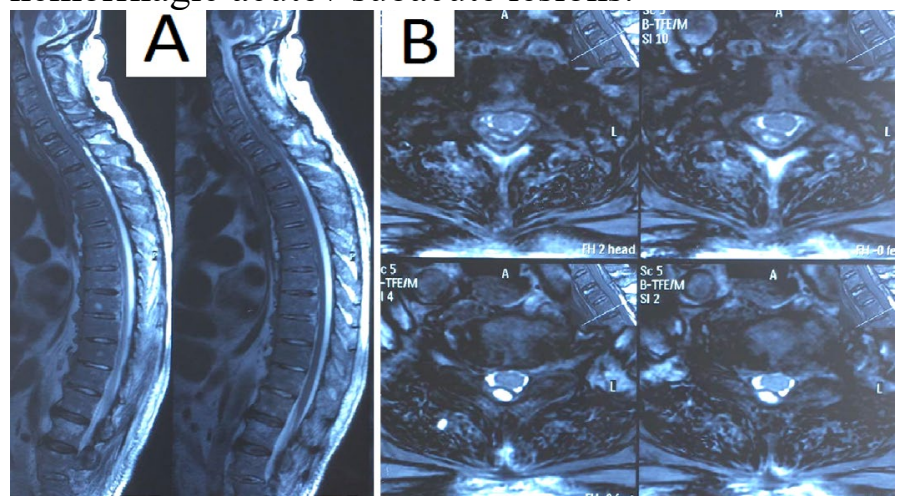

Fig.3 Pre-surgical MRI imagery.(A) Sagittal T2-weighted images: posterior epidural area of increased signal intensity (acute SEDH), compressing the spinal cord at $\mathrm{C} 3-\mathrm{Th} 2$ vertebral levels. (B) Transversal T2-weighted sections: right posterolateral SEDH.

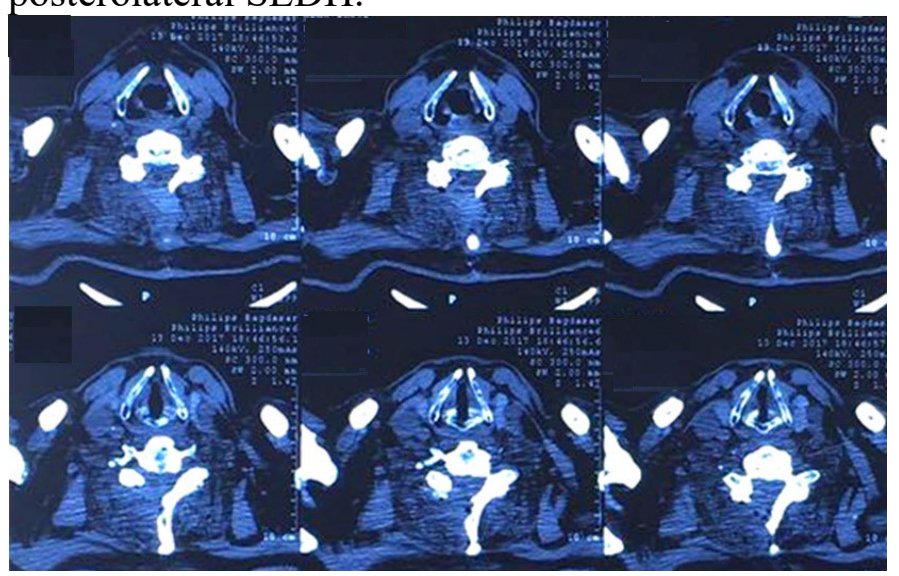

Fig.4 Post-surgical CT spine control, right hemilaminectomy.
He was transferred in the Neurorehabilitation Clinic on 29.01.2018 with severe post-surgical pain and depression. Clinical examination revealed normal vital signs: respiratory rate $-22 / \mathrm{min}$, chronic AF with a $74 \mathrm{bpm}$ heart rate, blood pressure $-140 / 90 \mathrm{mmHg}$, indwelling urinary catheter, post-operative wound dehiscence (healed per secundam, fig.6).
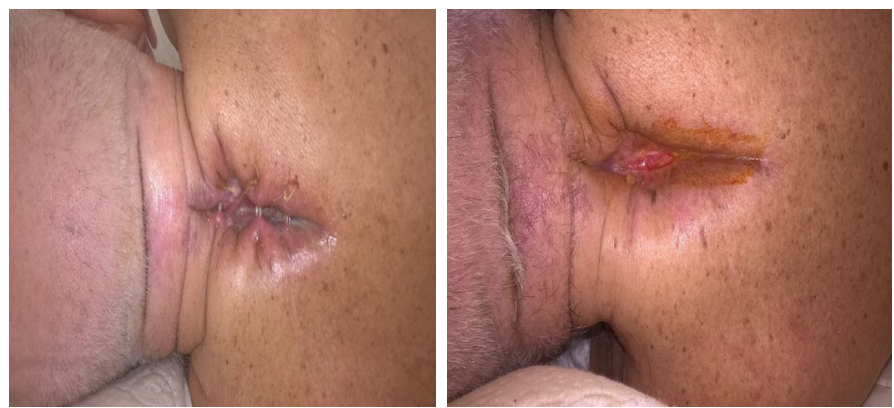

Fig.6 Post-operative wound, healed per secundam (left: at admission in the neurorehabilitation clinic on 31.01.2018; right: at discharge on 19.02.2018)

Neurological evaluation of the extent and severity of the spinal cord lesion, assessment of the rehabilitation targets and objectives, respectively the evolution were and quantified using the American Spinal Injury Association (ASIA) Impairment Scale (AIS).

Post-surgical neurological status at admission on rehabilitation was also C4 AIS-C tetraplegia: the global motor score was 50/100 (upper right limb 8/25, lower right limb 7/25; upper left limb 16/25; lower left limb 19/25): pin prick score was 100/112 (right side 49/56, respectively left side 51/56), and light touch score was $110 / 112$ (bilaterally 55/56).

Work-up. Usual blood tests revealed a slight anemia $\mathrm{Hgb} 9.7 \mathrm{~g} / \mathrm{dL}$ and slight leucocitosis WBC $12.71 \times 10^{3} / \mathrm{uL}$ (on 13.12.2017), then normalized (on 30.01.2018). Blood glucose, renal and liver panels were within normal parameters (estimated creatinine clearance was $58.53 \mathrm{ml} / \mathrm{min} 1.73 \mathrm{~m} 2$ ). Coagulation tests were influenced by the use of LMWH, eventually replaced with apixaban.

Post-surgical MRI control revealed the absence of the SEDH, showing only the $\mathrm{C} 7$ vertebral fracture and centromedullary edema (extended about $3 \mathrm{~cm}$, fig.5). 


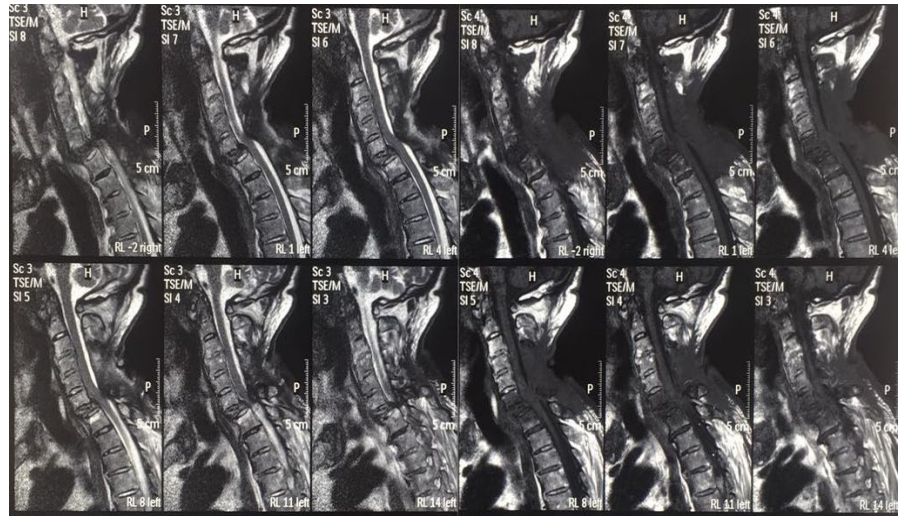

Fig.5 Post-surgical MRI control, sagittal T2weighted images (05.02.2018): C7 compression vertebral fracture and centromedullary edema.

The rehabilitation program was individualized and adapted to the current clinical-biological status; the objectives and methods, physical and occupational therapy approaches were focused on improvement of ADL abilities, cardio-respiratory endurance, bladder training program and avoiding complications.

Medication consisted in a synergic association of vitamins, neurotrophic supplements, analgesics, antidepressants, urinary disinfectants, and antibiotics.

During admission in the surgical ward the vitamin $\mathrm{K}$ antagonist (VKA) acenocumarol was replaced with a low-molecular-weight heparin (LMWH) Nadroparine /Fraxiparine 6000 U, subcutaneously.

After a careful analysis of risks and benefits, considering CHADS2VASc $=6$ as a high risk score for stroke in our patient with AF [20], and Has-Bled Score $=6$ as a major high bleeding risk [21], we reconsidered the injectable anti-factor Xa (LMWH), and prescribed a novel / direct oral anticoagulant (apixabanum, $2 \times 2.5 \mathrm{mg}$ BID) for a "long cruise" anticoagulant therapy, to prevent AFrelated stroke.

The subject had a favorable neurological evolution and was discharged as C7 AIS-D tetraplegia (global motor score 81/100: upper right limb $14 / 25$, lower right limb 20/25 ; upper left limb 22/25; lower left limb 25/25); pin prick score was 103 (right side 49/56, respectively left side 54/56), and light touch score was $112 / 112$.

At discharge he was capable to walk a few steps, with lateral assistance and support. He was recommended a wheel chair and sterile pre-lubricated
Nelaton catheters, for intermittent urinary program of bladder rehabilitation (both items being freely provided by the National Insurance House).

Discussion: Spinal epidural hematoma $(\mathrm{SEDH})$ is a rare but serious condition. Old age, hypertension, and anticoagulant therapy are the main risk factors for SEDH. Any age groups can be affected, but most cases were in their $60 \mathrm{~s}$ or $70 \mathrm{~s}$, with a slight predominance of the masculine gender [12], [22].

Summing data published between 20042016 one identified about 700 cases [8]. A new personal literature search found another 35 papers describing 40 new case reports published in 2017, respectively 8 case reports published in 2018 .

Messerer (2012) [10] proposed a practical classification of the spinal epidural hematomas according to the most probable etiology (whatever the associated factors), in six groups: spontaneous, secondary, iatrogenic, traumatic, recurrent, and idiopathic. Spontaneous hematoma is most often defined as a condition occurring in the absence of any traumatic event or iatrogenic procedure.

The present case has some clinical problems to be considered. Its main particularities consist in puzzled etiopathogenetic mechanisms and the difficulty to accurately indicate the chronological chain of events that generated the acute SEDH.

Literature data emphasize that etiology remains unclear in approximately $40 \%$ (up to 50 $75 \%$ ) cases of epidural hematoma, [3], [10], [17]. In our subject the exact underlying etiopathogenetic mechanism remained uncertain.

The patient was chronically anticoagulated with a VKA (acenocumarol), and daily ingested alcohol (alcohol consumption representing a factor incriminated in INR modification). Data referring to a retrospective estimation of how long the patient was efficiently anticoagulated (therapeutic time in range, TTR\%) were not available.

Main risk factors incriminated as iatrogenic causes for a "spontaneous" epidural hematoma are: poorly managed arterial hypertension [14], [15], [16], anticoagulant therapy (warfarin [22], [23], acenocumarol or even novel anticoagulants such as rivaroxaban [16], [24], [25]), or in very rare situations, low-dose aspirin (75-100 $\mathrm{mg}$ daily) [8], [14], [26], [27] and/ or other antiplatelet medication (clopidogrel [26], [28]). 
Spontaneous SEDH is a rare cause of spinal cord compression in adults, especially in the elderly [27]. During the acute neurosurgical episode, differential diagnosis was made with other epidural space-occupying lesions: traumatic disc hernia, other hematomas associated with congenital or acquired vascular lesions (arteriovenous spinal malformations), hemorrhagic tumors, hemophilia.

Typically an epidural hematoma is preceded / accompanied by local pain in the spine, [3], [9], [10]. Sometimes spontaneous cervical epidural hematoma is not always an obvious diagnosis [10], because in some elderly patients, back or neck pain might be absent [29]. In our case, the typical sequential evolutive paradigm (characterized by sudden onset with spinal radicular pain, followed by progressive neurological deficits due to spinal cord compression) was absent.

Accumulation of blood in the epidural space compressed the spinal cord and generated neurologic deficits. Added to the pre-existent congenital feet disability, these impairments predisposed to samelevel fall and subsequently to vertebral fracture.

Falls are the leading mechanism of injury for patients older than age 65 , elderly being predisposed to same-level fall (cause of injury, disability, and even death), resulting in cumulated chronic health problems [30], [31], [32]. Predisposing risk factors include: increasing age, gender $(78 \%$ male predominance), chronic alcoholism, vertebral degeneration, polypragmasia, sensory deficits, comorbidities, disability of the lower extremities, foot problems, cognitive impairment [32], [33], [34]. Cervical spine fractures due to ground level fall are relatively common in the elderly [35].

The physiopathology of our patient's SEDH was probably mixed - iatrogenic and traumatic, consequence of the cervical spine fracture, after ground to floor low-intensity biomechanical impact.

Another particularity of this case report is represented by the type and level of the vertebral fracture. The spinal fracture type is explained by the biomechanical dynamic proprieties of the head. The vertebral injury incurred is determined predominantly by the force vector applied during impact and the intrinsic strength and anatomy of $\mathrm{C} 2$ vertebral body and its surrounding spinal elements [36]. In neutral, vertical position, the average weight of human head is around 4.5 to $5 \mathrm{~kg}$ at rest. Our patient had accidentally fallen on ischial tuberosities and then on his back, without cranial impact with the surrounding objects (e.g. furniture). Typically, this type of level fall causes fractures of the atlanto-axial vertebral complex (C1-2), different from the current reported case (C7 compression vertebral fracture). As the head is bending forward, its weigh increases from $13 \mathrm{~kg}$ (at $\left.15^{\circ}\right)$, to $20 \mathrm{~kg}\left(\right.$ at $\left.30^{\circ}\right), 24,5 \mathrm{~kg}\left(\right.$ at $\left.45^{\circ}\right), 30 \mathrm{~kg}$ (at $60^{\circ}$ ) [37].This biomechanical variables might explain the structural wedge deformity of the $\mathrm{C} 7$ vertebral fracture compression in our patient (fig.5).

Another puzzling clinical aspect is represented by the short episode of loss of consciousness after falling, possibly owed to a cardiogenic syncope (chronic AF, hypodiastolic phenomena with global cerebral circulatory insufficiency) or to a transient ischemic attack.

Due to the severe cardiac pathology, the surgical decompressive intervention was postponed (for about two weeks); although surgery was performed during the early sub-acute phase, our patient had a good neurologic evolution during rehabilitation.

Despite the immediate good neurologic evolution during rehabilitation, the patient's functional prognosis might be poor, due to the advanced age and complex association of disabilities: the severe cardiovascular pathology and the disturbances of the neuro-myo-atrokinetic apparatus. Somatic Body functions and structural impairments at discharge consisted in: previous disabling congenital biomechanical feet condition, the poor hand prehension capacity, impeding a safe utilization of the walking frame or crutches, and incomplete recovery of force and coordination in the lower limbs. This health-related precarious condition had severe repercussions on the subject's Activity (related to tasks and basic activities of daily living) and Participation, affecting the outcome of rehabilitation, and his quality of life.

Our patient's vital prognosis depends on prevention of most common complications encountered in SCI (such as urinary tract infections, bowel problems, pressure sores, pulmonary infections) [38], in the context of the associated severe cardiac and neurological co-morbidities.

At discharge he was recommended apixabanum as a "long cruise" anticoagulant therapy, in order to provide secondary and tertiary prophylaxis of thromboembolic events (mainly cardio-embolic stroke), as well to diminish the hemorrhagic risk 
(nevraxial recurrence or other general severe bleeding). Due to the favourable pharmacokinetic properties (simple BID administration in fixed doses, low inter and / or intra-subject variability, multiple elimination pathways, not requiring laboratory monitoring - all important aspects for boosting compliance [39], [40], [41]) the patient had a good psychological adherence to treatment.

Conclusions Clinicians should consider the remote risk of SEDH (even with atypical clinical presentation) in patients with $\mathrm{AF}$ and anticoagulant medication. The clinical case underlies the importance of an individualized therapeutic approach (after careful assessment of benefits against risks, efficacy and safety profile).

Despite a postponed decompressive intervention (imposed by the severe comorbidities), our patient neurologically improved without recurrence, following a complex neurorehabilitation program.

Conflict of interest: Authors have no conflicts of interest to declare.

The paper has the approval of the Ethical Committee of the Hospital.

Written consent was obtained from the patient and next of kin.

\section{References}

1. Holtas S, Heiling M, Lönntoft M, Spontaneous spinal epidural hematoma: findings at MR imaging and clinical correlation, Radiology, 1996, 199(2), 409-13

2. Figueroa J, DeVine JG, Spontaneous spinal epidural hematoma: literature review, Journal of Spine Surgery, 2017, 3(1), 58-63

3. Duarte MP, da Silva LCL, de Sousa B G, Mallard SP, Agra CG. Sudden back pain as clinical presentation of spontaneous spinal epidural hematoma. Arq. Neuro-Psiquiatr, 2017; 75(1): 76-76

4. Kishen T, Etherington G, Diwan A, Cranial subdural haematoma with concomitant spinal epidural and spinal subarachnoid haematomas: a case report, Journal of Orthopaedic Surgery, 2009, 17(3), 358-60

5. Raj R, Seppälä M, Siironen J, Spontaneous Spinal Epidural Hematoma: A Surgical Case Series of Ten Patients. World Neurosurg, 2016;93:55-9

6. Fedor M, Kim E, Ding K, Muizelaar P, Kim K, Spontaneous Spinal Epidural Hematoma: A Retrospective Study on Prognostic Factors and Review of the Literature. Korean J Spine, 2011 8(4): 272-282
7. Akimoto T, Yamada T, Shinoda S, Asano Y, Nagata D, Spontaneous Spinal Epidural Hematoma as a Potentially Important Stroke Mimic, Journal of Central Nervous System Disease, 2014, 6, 15-20

8. Anghelescu A, Rasina A, A case of spontaneous thoracic spinal epidural haematoma (triggered by weight lifting, at a professional sportsman), with good outcome after surgical decompression and neurorehabilitation, Spinal Cord Series and Cases, 2017. doi:10.1038/scsandc. 2017.1

9. Mendes PD, Lopes CL, Baptista GS, Scaldaferri PM, Cariri GA, Sudden back pain as clinical presentation of spontaneous spinal epidural hematoma, Arq Neuropsiquiatr, 2017; 75(1):76.

10. Messerer M, Dubourg J, Diabira S, Robert T, Hamlat A, Spinal epidural hematoma: not always an obvious diagnosis, Eur J Emerg Med., 2012.19(1), 2-8

11. Raasck K, Habis A, Aoude A, Simões L, Barros F, Reindl R, et al., Spontaneous spinal epidural hematoma management: a case series and literature review, Spinal Cord Series and Cases, 2017. 3, 16043; doi:10.1038/scsandc.2016.43,

12. Kreppel D, Antoniadis G, Seeling W, Spinal hematoma: a literature survey with meta-analysis of 613 patients. Neurosurg Rev. 2003; 26(1):1-49.

13. Groen RJ, Non-operative treatment of spontaneous spinal epidural hematomas: a review of the literature and a comparison with operative cases, Acta Neurochir (Wien), 2004 146(2), 103-10.

14. Kim KT, Cho DC, Ahn SW, Kang SH, Epidural Hematoma Related with Low-Dose Aspirin : Complete Recovery without Surgical Treatment. $J$ Korean Neurosurg Soc. 2012; 51(5):308-11.

15. Tawk C, El Hajj Moussa M, Zgheib R, Nohra G, Spontaneous epidural hematoma of the spine associated with oral anticoagulants: 3 Case Studies, Int J Surg Case Rep. 2015;13:8-11

16. Ozel O, Demircay E, Kircelli A, Cansever T, Atypical Presentation of an Epidural Hematoma in a Patient Receiving Rivaroxaban After Total Hip Arthroplasty. Orthopedics. 2016;1;39(3): 558-60.

17. Buchanan CC, Lu DC, Buchanan C, Tran TT. Spontaneous spinal epidural hematoma and spinal cord infarction following orthotopic liver transplantation: Case report and review of the literature. Surg Neurol Int. 2013, 29;4 (Suppl 5):S359-61.

18. Dziedzic T, Kunert P, Krych P, Marchel A, Management and neurological outcome of spontaneous spinal epidural hematoma. $J$ Clin Neurosci. 2015; 22(4):726

19. Muñoz Gonzáleza A, Cuelloa JP, Rodríguez Cruza, PM, Iglesias Mohedanoa AM, Domínguez Rubio FR, Romero Delgado A, et al., Spontaneous spinal 
epidural haematoma: A retrospective study of a series of 13 cases, Neurologia, 2015; 30, 393-400

20. CHADS2VASc

https://qxmd.com/calculate/calculator 41/cha2ds2vasc-score-for-af]

21. Has-Bled Score http://www.mdcalc.com/has-bledscore-for-major-bleeding-risk/

22. Sandvig A, Jonsson H, Spontaneous chronic epidural hematoma in the lumbar spine associated with Warfarin intake: a case report. Springerplus. 2016 21;5(1):1832. eCollection 2016

23. Yabe H, Ishii A, Niikawa N, Matsubayashi H, Kakei M, Kawakami M, Sugawara H. - An elderly patient who developed spontaneous spinal epidural hematoma during warfarin therapy. Intern Med. 2012;51(11):1429-32

24. Castillo JM, Afanador HF, Manjarrez E, Morales XA, Non-Traumatic Spontaneous Spinal Subdural Hematoma in a Patient with Non-Valvular Atrial Fibrillation During Treatment with Rivaroxaban. Am J Case Rep. 2015, 19;16:377-81. doi: 10.12659/AJCR.893320

25. Ismail R, Zaghrini E, Hitti E. Spontaneous Spinal Epidural Hematoma in a Patient on Rivaroxaban: Case Report and Literature Review, J Emerg Med. 2017;53(4):536-539.

10.1016/j.jemermed.2017.06.005

26. Wang L, Liu G, Subramaniam S, Wong TA, Kumar N. Spontaneous spinal epidural haematoma after antiplatelet treatment: a report of two cases. J Orthop Surg (Hong Kong). 2012; 20(3):386-90

27. Dimou J, Jithoo R, Morokoff A, Spontaneous spinal epidural haematoma in a geriatric patient on aspirin. $J \quad$ Clin Neurosci. 2010; 17(1):142-4. doi: 10.1016/j.jocn.2009.03.021.

28. Bhat KJ, Kapoor S, Watali YZ, Sharma JR, Spontaneous epidural hematoma of spine associated with clopidogrel: A case study and review of the literature. Asian J Neurosurg 2015;10:54

29. Hongo T, Iseda K, Tsuchiya M, Inaba M, Nozaki S., Takahashi K., ... Fujiwara, T. Two cases of spontaneous cervical epidural hematoma without back or neck pain in elderly Japanese men. Acute Medicine \& Surgery, 2018,5(2),181-184 http://doi.org/10.1002/ams2.317

30. Bank M, Gibbs, K, Sison C, Kutub N, Paptheodorou A, Lee S, ...Bloom O, Age and Other Risk Factors Influencing Long-Term Mortality in Patients With Traumatic Cervical Spine Fracture. Geriatric Orthopaedic Surgery \& Rehabilitation, 2018, 9, 2151459318770882.

http://doi.org/10.1177/2151459318770882

31. Toda M, Nakatani E, Omae K, Fukushima M, Chin T, Age-specific characterization of spinal cord injuries over a 19-year period at a Japanese rehabilitation center. Jan Y-K, ed. PLoS ONE. 2018;13(3):e0195120.

doi:10.1371/journal.pone.0195120.

32. Anghelescu A, Clinical and pathophysiological considerations of gait limitations and high prevalence of falls, in elderly with most common, disabling neurological diseases, Int J Neurorehabilitation Eng, 2017, 4:2 DOI: 10.4172/2376-0281.1000262

33. Tinetti ME, Speechley M, Ginter SF, Risk factors for falls among elderly persons living in the community. N Engl J Med.1988 29;319(26):1701-7] .

34. Hester AL, Wei F, Falls in the community: state of the science. Clinical Interventions in Aging. 2013; 8:675-679. doi:10.2147/CIA.S44996]

35. Wang, H, Coppola M, Robinson RD, Scribner JT, Vithalani V, de Moor CE,...Delaney KA, Geriatric Trauma Patients With Cervical Spine Fractures due to Ground Level Fall: Five Years Experience in a Level One Trauma Center. Journal of Clinical Medicine Research.

2013;5(2):75-83. doi:10.4021/jocmr $1227 \mathrm{w}$

36. Benzel EC, Hart BL, Ball PA, Baldwin NG, Orrison WW, Espinosa M, Fractures of the C-2 vertebral body. J Neurosurg. 1994;81(2):206-12.

37. https://www.movementandcreativity.com/blog/podc ast/the-weight-of-your-head

38. Onose G, Anghelescu A, et al. Rehabilitation in conditions following spinal cord injuries, in Textbook of Neurosurgery [chapter in romanian: "Reabilitare in suferinte dupa traumatisme vertebro-medulare" in Tratat de Neurochirurgie, vol. 2], 2011 Editura Medicala (ISBN 978 - 973-39-0720

39. Trkulja V, Safety of apixaban for venous thromboembolism prophylaxis: the evidence to date, Drug Healthc Patient Saf, 2016, 18; 8, pp. 25-38.

40. Toth PP, Considerations for long-term anticoagulant therapy in patients with venous thromboembolism in the novel oral anticoagulant era, Vasc Health Risk Manag, 2016, 10; 12, 23-34.

41. Anghelescu A, Secondary prevention of systemic arterial and recurrent venous thromboembolic events, through extended time anticoagulation with apixaban, in an elderly patient with multiple comorbidities, clinical case, International Archives of Medicine, Section: Cardiology, 2016, 9, 164, doi: $10.3823 / 2035$. 